\title{
Magnetic Orders in Compounds Made of Icosahedral Rare-Earth Clusters
}

\author{
Takanobu Hiroto, Takeru Sato, Yuji Muro*, Hiroshi Yaguchi ${ }^{\dagger}$, \\ Toshiro Takabakate ${ }^{*}$, Ryuji Tamura \\ Department of Materials Science and Technology, Tokyo University of Science, Noda, Chiba 278-8510, Japan \\ Fax: 81-04-7124-1501, e-mail: takanobu.hiroto@gmail.com \\ *Department of Quantum Matter, AdSM, Hiroshima University, Higashi-Hiroshima, Hiroshima 739-8530, Japan \\ ${ }^{\dagger}$ Department of Physics, Tokyo University of Science, Noda, Chiba, Japan \\ ${ }^{\ddagger}$ Institute for Advanced Materials Research, Hiroshima University, Higashi-Hiroshima, Hiroshima 739-8530, Japan
}

We report magnetic properties of $\mathrm{Cd}_{6} \mathrm{RE}(\mathrm{RE}=\mathrm{Tb}, \mathrm{Sm})$ single-grained crystalline approximants. Magnetic susceptibilities show that various long-range magnetic orders occur at low temperatures for the compounds made of $\mathrm{RE}$ icosahedra: $\mathrm{Cd}_{6} \mathrm{~Tb}$ shows three types of antiferromagnetic orders with temperature and $\mathrm{Cd}_{6} \mathrm{Sm}$ exhibits also three types of magnetic orders with temperature, i.e., one antiferromagnetic and two types of ferrimagnetic orders.

Key words: Rare-Earth Compounds, Quasicrystalline Approximants, Magnetic Properties

\section{INTRODUCTION}

Quasicrystals $^{[1]}$ are known as aperiodic solids that lack translational symmetry but have rotational symmetries forbidden in crystals, i.e., fivefold, eightfold, tenfold and 12-fold rotational symmetries. Until now, a number of quasicrystals have been discovered over 70 alloy systems. However, because of the existence of chemical disorder which are thought to obscure "quasiperiodicity", it has been difficult to understand the structural and physical properties intrinsic to quasicrystals. In this respect, the Cd-based binary quasicrystals $\mathrm{Cd}_{5.7} \mathrm{Ca}^{[2]}$ and $\mathrm{Cd}_{5.7} \mathrm{Yb}^{[3]}$ which were discovered by Tsai et.al. in 2000 have helped us to study the structural and physical properties of quasicrystals since they have no chemical disorder. For an instance, the first successful structural analysis was performed in $\mathrm{Cd}_{5.7} \mathrm{Yb}^{[4]}$.

Amongst various physical properties of quasicrystals, the magnetic properties of quasicrystals containing $4 f$ rare earth elements have caught much attention. Magnetic measurements on the $\mathrm{Cd}(\mathrm{Zn})-\mathrm{Mg}-\mathrm{RE}$ ( $\mathrm{RE}=$ rare earth elements) quasicrystals have been performed systematically, which has shown spin glass-like behaviors of localized $\mathrm{RE}^{3+}$ spins at low temperature in all the cases, however, no long-range magnetic states has been reported ${ }^{[5,6,7]}$.

Meanwhile, crystalline approximants are also of significant interest since they allow us to understand the structural and physical properties of the quasicrystals with respect to the common local structure. The $\mathrm{Cd}_{6} \mathrm{M}$ ( $\mathrm{M}=\mathrm{Ca}, \mathrm{Y}$, Rare earth elements) compounds, body-centered-cubic (bcc) crystals with space group Im-3 and $\mathrm{a} \sim 1.5 \mathrm{~nm}^{[8]}$, are known as crystalline approximants to the $\mathrm{Cd}_{5.7} \mathrm{Ca}$ and $\mathrm{Cd}_{5.7} \mathrm{Yb}$ icosahedral quasicrystals. According to the single crystal structural analyses ${ }^{[9,10]}$, the $\mathrm{Cd}_{6} \mathrm{M}$ compounds contain 168 atoms in a unit cell and consists of four successive shells (called Tsai-type cluster, see Figure 1(a)), i.e., from the cluster center, a $\mathrm{Cd}_{4}$ tetrahedron, a $\mathrm{Cd}_{20}$ dodecahedron, a $\mathrm{M}_{12}$ icosahedron and a $\mathrm{Cd}_{30}$ icosidodecahedron, together with "glue" $\mathrm{Cd}$ atoms which fill the space in between the clusters. It is noted that $\mathrm{M}$ atoms occupy the icosahedral $(24 \mathrm{~g})$ site without chemical or positional disorder. The systems can also be regarded as new $4 f$ spin systems that are composed of icosahedral $\mathrm{RE}_{12}$ clusters (See Figure1 (b)).

In this article, we report results of magnetic property measurements on $\mathrm{Cd}_{6} \mathrm{~Tb}$ and $\mathrm{Cd}_{6} \mathrm{Sm}$ as typical examples

(a)

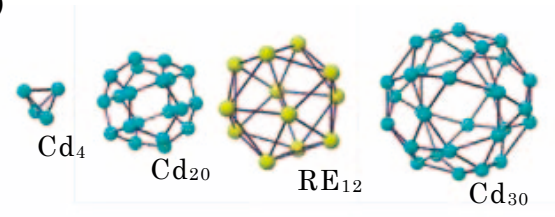

(b)

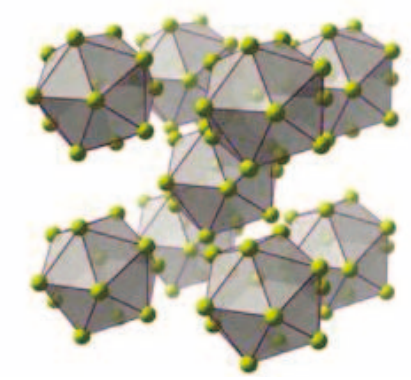

Figure1. (a) Tsai-type cluster and (b) $b c c$ packing of $\mathrm{RE}_{12}$ icosahedra in $\mathrm{Cd}_{6} \mathrm{RE}$ cubic approximants. 
and discuss their magnetic properties as well as their long-range magnetic orders at low temperatures.

\section{EXPERIMENTAL PROCEDURES}

Single grains of $\mathrm{Cd}_{6} \mathrm{RE}(\mathrm{RE}=\mathrm{Tb}, \mathrm{Sm})$ approximants were prepared by a self-flux growth technique. Pure elements of the starting composition of $\operatorname{Cd}(6 \mathrm{~N}): \operatorname{RE}(3 \mathrm{~N})$ $=9: 1$ were placed inside an alumina crucible sealed inside a quartz tube under Argon atmosphere. The raw materials were melted at $993 \mathrm{~K}$ for $24 \mathrm{~h}$, and slowly cooled at the rate of $-2 \mathrm{~K} / \mathrm{h}$ to $773 \mathrm{~K}$. At $773 \mathrm{~K}$, the crucible was taken out from the muffle furnace and the $\mathrm{Cd}$ melt was rapidly decanted by turning the crucible upside down and centrifuging using a high-speed centrifuge. The obtained single grains exhibit well defined facets surrounded by $\{100\}$ and $\{110\}$ planes. Single grains were subsequently annealed in order to improve the sample homogeneity as well as to reduce point defects. The magnetic properties were measured from 1.8 to $300 \mathrm{~K}$ by using a SQUID magnet meter (Quantum Design, MPMS) under 50 or 1000 Oe.

\section{RESULTS AND DISCUSSION}

3.1 Magnetic properties at high temperatures

Figures 2(a) and (b) show the magnetic susceptibility $\chi$ and the inversed magnetic susceptibility $1 / \chi$ as a function of temperature measured from 2 to $300 \mathrm{~K}$ for $\mathrm{Cd}_{6} \mathrm{~Tb}$ and $\mathrm{Cd}_{6} \mathrm{Sm}$ approximants, respectively. The susceptibility of $\mathrm{Cd}_{6} \mathrm{~Tb}$ above $50 \mathrm{~K}$ obeys the CurieWeiss law, $\chi(T)=\frac{N_{A} \mu_{e f f}{ }^{2}}{3 k_{B}(T-\theta)}$, where, $N_{A}$ is the Avogadro's number, $\mu_{\text {eff }}$ is the effective magnetic moment, $k_{B}$ is the Boltzmann's constant and $\Theta$ is the Curie-Weiss temperature, respectively. On the other hand, the magnetic susceptibility of $\mathrm{Cd}_{6} \mathrm{Sm}$ (Figure 2(b)) does not obey the Curie-Weiss law, most likely because of the small energy difference between the Hund's rule ground state $(\mathrm{J}=5 / 2)$ and the first excited state $(\mathrm{J}=7 / 2)^{[11]}$. Therefore, the susceptibility data of $\mathrm{Cd}_{6} \mathrm{Sm}$ were fitted by a following formula which incorporates a temperature-independent Van Vleck term due to the thermal excitation of first excited state into the Curie-Weis law, $\chi(T)=\left(\frac{N_{A}}{k_{B}}\right)\left[\frac{\mu_{e f f}^{2}}{3(T-\theta)}+\frac{\mu_{B}}{\delta}\right]$,where, $\mu_{B}$ is the Bohr magneton and $\delta=7 \Delta / 20$ where $\Delta$ is the energy difference in units of Kelvin between the $\mathrm{J}=5 / 2$ and $\mathrm{J}=7 / 2$ multiplet states ${ }^{[12]}$. Table 1 shows the parameters obtained by the least square fits to the magnetic susceptibilities of $\mathrm{Cd}_{6} \mathrm{~Tb}$ and $\mathrm{Cd}_{6} \mathrm{Sm}$. The negative $\Theta$ values in both systems mean that major interaction between $4 f$ spins are antiferromagnetic in the paramagnetic state. The values of effective magnetic moments are approximately the same as those of free $\mathrm{RE}^{3+}$ ions, which suggests that $\mathrm{RE}^{3+}$ atoms are well (a)
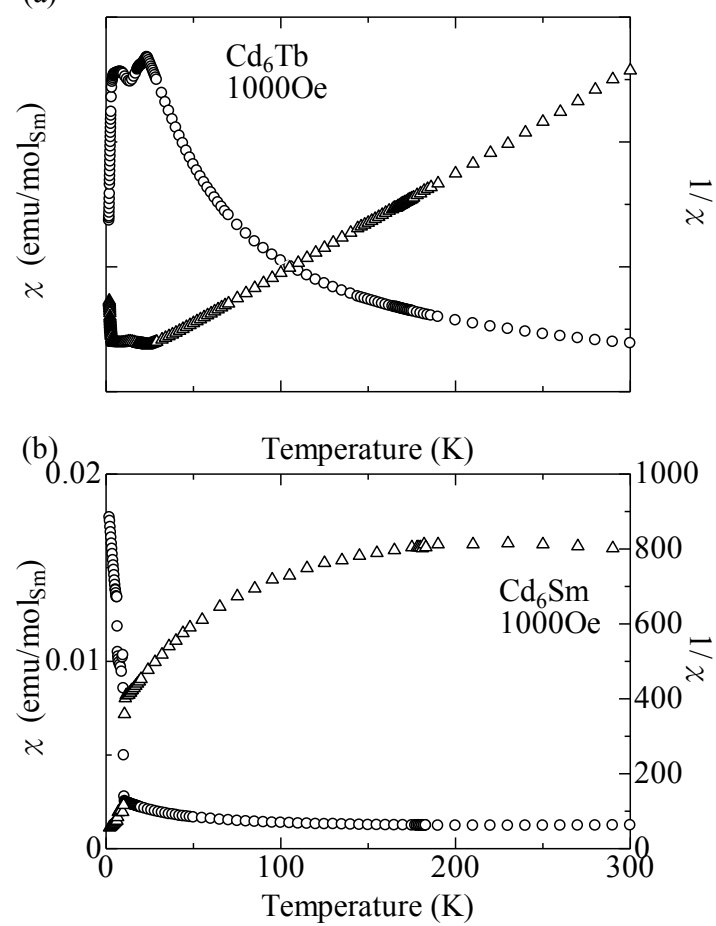

Figure 2. Magnetic susceptibility (open circle) and inversed susceptibility (open triangle) of (a) $\mathrm{Cd}_{6} \mathrm{~Tb}$ and (b) $\mathrm{Cd}_{6} \mathrm{Sm}$ approximants measured in a field of 1000 Oe

localized at the vertices of the $\mathrm{RE}_{12}$ icosahedron, hardly affected by crystalline-field splitting effect.

3.2 Magnetic orders at low temperatures

Figures 3(a) and (b) show low-temperature magnetic susceptibility as a function of temperature from 1.8 to 30 $\mathrm{K}$ for $\mathrm{Cd}_{6} \mathrm{~Tb}$ and $\mathrm{Cd}_{6} \mathrm{Sm}$ approximants, respectively. The susceptibility of $\mathrm{Cd}_{6} \mathrm{~Tb}$ exhibits three successive anomalies at 1.9, 19.0 and $24.0 \mathrm{~K}$ which are interpreted as antiferromagnetic transitions. The result is consistent with those on a poly-grain sample recently reported by Tamura et. al. ${ }^{[13]}$ The $\mathrm{Cd}_{6} \mathrm{Sm}$ approximant also exhibits three successive magnetic transitions with temperature, i.e., an antiferromagnetic one $(12.5 \mathrm{~K})$ and two ferrimagnetic ones $(10.0$ and $6.5 \mathrm{~K})$. For both

Table 1. Weiss temperaturse $\Theta$ and effective magnetic moments $\mu_{\text {eff }}$ of $\mathrm{Cd}_{6} \mathrm{~Tb}$ and $\mathrm{Cd}_{6} \mathrm{Sm}$. For a comparison, the magnitude of the theoretical $\mathrm{RE}^{3+}$ moments $\left(\mu_{\text {theol. }}\right)$ are also shown.

\begin{tabular}{c|cc}
\hline & $\mathrm{Cd}_{6} \mathrm{~Tb}$ & $\mathrm{Cd}_{6} \mathrm{Sm}$ \\
\hline$\Theta / \mathrm{K}$ & -17.5 & -16 \\
\hline$\mu_{\text {eff }} / \mu_{\mathrm{B}}$ & 9.95 & 0.59 \\
\hline$\mu_{\text {theol. }} / \mu_{\mathrm{B}}$ & 9.72 & 0.85 \\
\hline
\end{tabular}


(a)

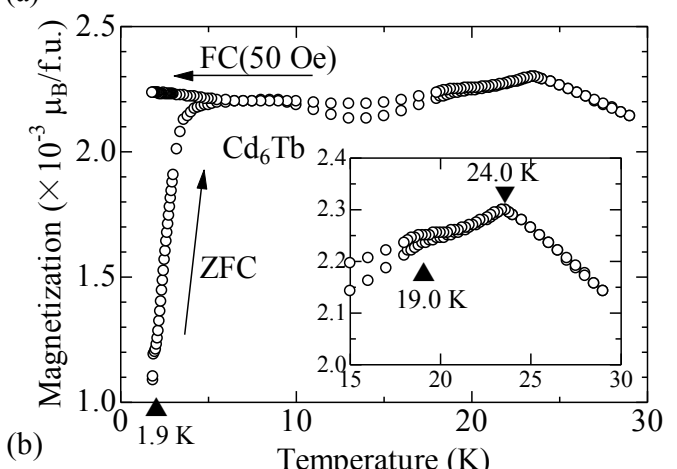

(b)

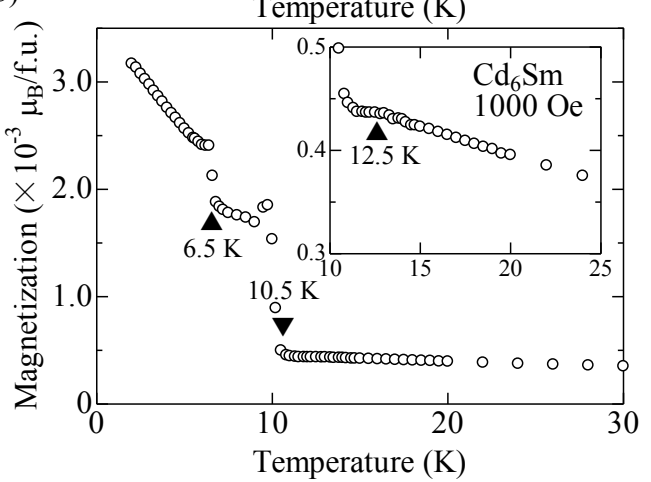

Figure 3. Low temperature magnetic susceptibility of (a) $\mathrm{Cd}_{6} \mathrm{~Tb}$ and (b) $\mathrm{Cd}_{6} \mathrm{Sm} . \mathrm{Cd}_{6} \mathrm{~Tb}$ was measured at zero-field cooled (ZFC) and field cooled (FC) under 50 Oe. $\mathrm{Cd}_{6} \mathrm{Sm}$ was measured under 1000 Oe.

systems, specific heat also displays anomalies and/or peaks at the transition temperatures (not shown) indicating that the successive transitions are due to long-range magnetic orders. These results in $\mathrm{Cd}_{6} \mathrm{RE}$ are different from the spin glass-like behaviors observed in, for instance, Ag-In-RE approximants ${ }^{[14]}$. The present work clearly shows that long-range magnetic orders are allowed for $4 f$ magnetic moments sitting on the vertices of an icosahedron.

\section{CONCLUSION}

We have measured magnetic properties of $\mathrm{Cd}_{6} \mathrm{RE}$ $(\mathrm{RE}=\mathrm{Tb}, \mathrm{Sm})$ single-grained crystalline approximants. Above $50 \mathrm{~K}$, the magnetic susceptibility of $\mathrm{Cd}_{6} \mathrm{~Tb}$ obeys the Curie-Weiss law whereas that of $\mathrm{Cd}_{6} \mathrm{Sm}$ does not follow the law but is explained by including the contribution from the van Vleck term. The obtained effective magnetic moments suggest that $\mathrm{RE}^{3+}$ atoms are well localized in a trivalent state at the vertices of the $\mathrm{RE}_{12}$ icosahedron. At low temperatures, both the approximants exhibit successive magnetic transitions, i.e., antiferro- and/or ferri- magnetic orders, below $30 \mathrm{~K}$. These results are different from the observations in other quasicrystals and approximants which exclusively show spin glass-like behaviors.

\section{REFERRENCES}

[1] D. Shechtman, I. Blech, D. Gratias and J. W. Cahn, Phys. Rev. Lett. 53, 1951-1953 (1984).

[2] A. P. Tsai, J. Q. Guo, E. Abe, H. Takakura and T. J. Sato, Nature 408, 537-538 (2000).

[3] J. Q. Guo, E. Abe and A. P. Tsai, Phys. Rev. B 62, R14605-R14608 (2000).

[4] H.Takakura, C. P. Gómez, A. Yamamoto, M. de Boissieu and A. P. Tsai, Nature Materials 658 (2007).

[5] T. J. Sato, H. Takakura and A. P. Tsai, Phys. Rev. B 61, 476-486 (2000)

[6] T. J. Sato, J. Q. Guo and A. P. Tsai, J. Phys.: Condens. Matter 13, L105-L111 (2001)

[7] T. J. Sato. Acta Cryst. A61, 39-50 (2005)

[8] G. Bruzzone, M. L. Fornasini and F. Merlo, J. Less. Common Met., 30, 361-375 (1973).

[9] C. P. Gómez and S. Lidin, Phys. Rev. B 68, 024203 (2003)

[10] S. Y. Piao, C. P. Gómez, and S. Lidin, Z. Naturforsch., B: Chem. Sci. 61b, 644 (2006).

[11] W. M. Yuhasz, N. A. Frederick, P. C. Ho, N. P. Butch, B. J. Taylor, T. A. Sayles and M. B. Maple, Phys. Rev. B 71, 104402 (2005)

[12] H. C. Hamaker, L. D. Woolf, H. B. MacKay, Z. Fisk, and M. B.Maple, Solid State Commun. 32, 289 (1979).

[13] R. Tamura, Y. Muro, T. Hiroto, K. Nishimoto, and T. Takabatake, Phys. Rev. B 82, 220201 (2010).

[14] S. Ibuka, K. Iida and T. J. Sato, J. Phys.: Condens. Matter 23, 056001 (2011)

(Received Janurary 31, 2011; Accepted May 17, 2009) 